\title{
Evaluation of different treatment strategies between right-sided and left-sided pneumonectomy for stage I-IIIA non-small cell lung cancer patients
}

Bo Jia

Beijing Cancer Hospital

Qiwen Zheng

Beijing Cancer Hospital

Jianjie Li

Beijing Cancer Hospital

Jun Zhao

Beijing Cancer Hospital

Meina Wu

Beijing Cancer Hospital

Tongtong An

Beijing Cancer Hospital

Yuyan Wang

Beijing Cancer Hospital

Minglei Zhuo

Beijing Cancer Hospital

Xue Yang

Beijing Cancer Hospital

Jia Zhong

Beijing Cancer Hospital

Hanxiao Chen

Beijing Cancer Hospital

Yujia Chi

Beijing Cancer Hospital

Jingjing Wang

Beijing Cancer Hospital

Xiaoyu Zhai

Beijing Cancer Hospital

ziping wang ( $\square$ wangzp2007@126.com )

Beijing Cancer Hospital

Research article

Keywords: non-small cell lung cancer, left-side, right-side, pneumonectomy, treatment

Posted Date: March 31st, 2020

DOI: https://doi.org/10.21203/rs.3.rs-20041/v1

License: (c) (7) This work is licensed under a Creative Commons Attribution 4.0 International License. Read Full License 


\section{Abstract}

\section{Background}

This study aimed to assess the different survival outcome of stage I-IIIA NSCLC patients who received right-sided and left-sided pneumonectomy, and to further develop the most appropriate treatment strategies.

Methods

We accessed data from the Surveillance, Epidemiology, and End Results database in the United States for the present study. An innovative propensity score matching analysis was used to minimize the variance between groups.

Results

For 2,683 patients who received pneumonectomy, cancer-specific survival ( $\mathrm{HR}=0.863,95 \% \mathrm{Cl}$ : 0.771 to $0.965, \mathrm{P}=0.010)$ and overall survival (OS) (HR=0.875, 95\%Cl: 0.793 to $0.967, P=0.008)$ were significantly superior of left-sided pneumonectomy compared with right-sided pneumonectomy. Cancer-specific survival ( $\mathrm{HR}=0.847,95 \% \mathrm{Cl}: 0.745$ to $0.963, \mathrm{P}=0.011)$ and $\mathrm{OS}$ ( $\mathrm{HR}=0.858,95 \% \mathrm{Cl}: 0.768$ to $0.959, P=0.007$ ) were also significantly longer with left-sided over opposite-sided pneumonectomy after matching analysis for 2,050 patients. Adjuvant therapy could significantly prolong cancer-specific survival ( 67 versus 51 months, $\mathrm{HR}=1.314,95 \% \mathrm{Cl}$ : 1.093 to $1.579, \mathrm{P}=0.004$ ) and $\mathrm{OS}$ ( 46 versus 30 months, $\mathrm{HR}=1.458,95 \% \mathrm{Cl}: 1.239$ to $1.715, \mathrm{P}<0.001$ ) among left-sided pneumonectomy patients after matching procedure. While adjuvant therapy did not increase cancer-specific survival for right-sided pneumonectomy patients ( 46 versus 42 months, $\mathrm{HR}=1.112,95 \% \mathrm{Cl}$ : 0.933 to $1.325, \mathrm{P}=0.236$ ). Subgroup analysis showed that adjuvant chemotherapy could significantly improve cancer-specific survival and OS for all pneumonectomy patients. But radiotherapy was associated with worse survival for patients with right-sided pneumonectomy.

\section{Conclusions}

Pneumonectomy side could be deemed as an important factor when physicians choosing the most optimal treatment strategies. The abstract was presented at the 2018 American Society of Clinical Oncology Annual Meeting (Abstract \#8524)

\section{Introduction}

Lung cancer has been the most common cancer in China and globally [1, 2]. About 75-80\% lung cancer patients belong to nonsmall cell lung cancer (NSCLC) [3]. Pneumonectomy can approximately affect quality of life and long-term survival for several reasons. First, there are some complications of pneumonectomy including decreased respiratory function, bronchopleural fistulas, progressive pulmonary hypertension and elevated right-heart pressure, etc[4,5]. Second, it has been reported that patients received right or left-sided pneumonectomy may have different prognosis [6, 7], and patients with pneumonectomy in right lung were associated with higher incidence of mortality and morbidity than that in left side[8]. mainly due to the reason that right lung contributes more to overall lung function than that in the opposite side[9]. Third, the anatomical structure, blood supply and metastatic lymph node routes of bilateral lungs are not totally identical. Therefore, right and left-sided pneumonectomy may have distinctive long-term survival, but this problem has not been fully identified..

Although adjuvant chemotherapy has been the standard treatment for II-IIIA patients with surgery, few of them have examined patients undergoing pneumonectomy. Besides, the long-term risks and benefits of adjuvant radiotherapy after pneumonectomy remain controversial. It is of great value to illustrate the influence of adjuvant therapy for patients who received pneumonectomy. Therefore, the purpose of this study was to assess whether primary tumor sidedness could influence long-term survival in stage IIIIA NSCLC patients who received pneumonectomy, and to further develop the best treatment strategies for these patients.

\section{Patients And Methods}

\section{Data source}


We access data from the Surveillance, Epidemiology, and End Results (SEER) database in the United States for the present study[10]. Data codes were recorded by the North American Association of Central Cancer Registries (NAACCR)[11]. The 3rd edition of the International Classification of Diseases for Oncology (ICD-0-3) was used to code primary tumor histology..

\section{Cohort Selection}

NSCLC patients aged 18 years or older from the year 2004 to 2014 were included in this study. Patients with primary tumor location unknown were excluded. All patients received pneumonectomy. The study was exempted from ethical review by the Beijing Cancer Hospital. We obtained the data agreement and data files were downloaded directly from the SEER website[12]. Flow chart of patients' selection in this study was shown in eFigure1.

\section{Statistical analysis}

Patients were categorized into left-sided and right-sided pneumonectomy. We used chi-square test or Fisher's exact test between groups to perform univariate analysis. A propensity score was constructed to reduce potential bias. A multivariable logistic regression model was constructed including variables such as age, gender, race, year of diagnosis, tumor stage, $\mathrm{T}$ stage, $\mathrm{N}$ stage, original site, histology, number of lymph node dissection, radio therapy and chemotherapy. Matching (1:1) across the two groups was achieved with a 0.2-width caliper of the standard deviation of the logit of the propensity score. To evaluate the matching performance, baseline variables were compared using the McNemar's test between the matched groups, and a difference between - 0.1 and 0.1 is generally considered negligible to calculate standardized differences..

We assessed overall survival (OS) and cancer-specific survival using Kaplan-Meier curves in the matched groups. Relationships between pneumonectomy laterality and OS or cancer-specific survival was evaluated by Cox regression model stratified on matched pairs.

R software (version 3.3.3; http:// www.r-project.org) was used for statistical analysis. The significance levels were all two-sided, with statistical significance set at 0.05 .

\section{Results}

\section{Patients' characteristics}

In this study, 2,683 NSCLC patients with stage I-IIIA who received pneumonectomy between 2004 and 2014 were collected. In these patients, 1,150 patients received right-sided pneumonectomy and 1,533 patients with left-sided pneumonectomy. Patients' characteristics between the two groups were illustrated in Table 1. More patients with right-sided pneumonectomy patients had stage I $(P=0.006)$, N0 $(P=0.005)$, had overlapping lesion $(P=0.007)$, and had adenocarcinomas $(P<0.001)$. Less patients with pneumonectomy in right side received chemotherapy $(P=0.025)$. 
Table 1

Comparison of Baseline Characteristics Between Right-sided and Left-sided Pneumonectomy in the Original and Matched Data

Sets.

\begin{tabular}{|c|c|c|c|c|c|c|c|c|}
\hline \multirow[t]{2}{*}{ Variable } & \multicolumn{4}{|c|}{ Original Data Set } & \multicolumn{4}{|c|}{ Matched Data Set } \\
\hline & $\begin{array}{l}\text { Right-sided } \\
(n=1150)\end{array}$ & $\begin{array}{l}\text { Left-sided } \\
(n=1533)\end{array}$ & Sdiff & $P *$ & $\begin{array}{l}\text { Right-sided } \\
(n=1025)\end{array}$ & $\begin{array}{l}\text { Left-sided } \\
(n=1025)\end{array}$ & Sdiff & $\mathrm{Pt}$ \\
\hline \multicolumn{9}{|l|}{ Age } \\
\hline Up to 40 years & $17(1.5)$ & $13(0.8)$ & -0.059 & \multirow[t]{3}{*}{0.283} & $11(1.1)$ & $12(1.2)$ & -0.009 & \multirow[t]{3}{*}{0.748} \\
\hline 41 to 69 years & $862(75.0)$ & $1146(74.8)$ & -0.005 & & $775(75.6)$ & 788 (76.9) & -0.030 & \\
\hline $70+$ years & $271(23.6)$ & $374(24.4)$ & 0.019 & & $239(23.3)$ & $225(22.0)$ & 0.033 & \\
\hline \multicolumn{9}{|l|}{ Gender } \\
\hline Male & 792 (68.9) & $1020(66.5)$ & -0.050 & \multirow[t]{2}{*}{0.217} & 707 (69.0) & 703 (68.6) & 0.008 & \multirow[t]{2}{*}{0.882} \\
\hline Female & $358(31.1)$ & $513(33.5)$ & 0.050 & & $318(31.0)$ & $322(31.4)$ & -0.008 & \\
\hline \multicolumn{9}{|l|}{ Race/ethnicity } \\
\hline Caucasian & $990(86.1)$ & $1328(86.6)$ & 0.016 & \multirow[t]{4}{*}{0.251} & $893(87.1)$ & $887(86.5)$ & 0.017 & \multirow[t]{4}{*}{0.364} \\
\hline African-American & $97(8.4)$ & $128(8.3)$ & -0.003 & & $86(8.4)$ & $91(8.9)$ & -0.017 & \\
\hline Asian & $53(4.6)$ & $54(3.5)$ & -0.055 & & $37(3.6)$ & $39(3.8)$ & -0.010 & \\
\hline Other/Unknown & $10(0.9)$ & $23(1.5)$ & 0.058 & & $9(0.9)$ & $8(0.8)$ & 0.011 & \\
\hline \multicolumn{9}{|l|}{ Year of diagnosis } \\
\hline $2004-2007$ & $510(44.3)$ & $655(42.7)$ & -0.033 & \multirow[t]{3}{*}{0.694} & $453(44.2)$ & 429 (41.9) & 0.047 & \multirow[t]{3}{*}{0.196} \\
\hline $2008-2011$ & $400(34.8)$ & $545(35.6)$ & 0.016 & & $359(35.0)$ & 381 (37.2) & -0.045 & \\
\hline 2012-2014 & 240 (20.9) & 333 (21.7) & 0.021 & & $213(20.8)$ & 215 (21.0) & -0.005 & \\
\hline \multicolumn{9}{|l|}{ Tumor stage $\ddagger$} \\
\hline Stage I & $389(33.8)$ & $437(28.5)$ & -0.115 & \multirow[t]{3}{*}{0.006} & 330 (32.2) & $336(32.8)$ & -0.012 & \multirow[t]{3}{*}{0.148} \\
\hline Stage II & $403(35.0)$ & $613(40.0)$ & 0.102 & & $374(36.5)$ & $336(32.8)$ & 0.078 & \\
\hline Stage IIIA & $358(31.1)$ & $483(31.5)$ & 0.008 & & 321 (31.3) & $353(34.4)$ & -0.066 & \\
\hline \multicolumn{9}{|l|}{ Clinical T stage } \\
\hline T1 & 135 (11.7) & $161(10.5)$ & -0.039 & \multirow[t]{4}{*}{0.204} & $121(11.8)$ & 118 (11.5) & 0.009 & \multirow[t]{4}{*}{0.647} \\
\hline T2 & 797 (69.3) & $1100(71.8)$ & 0.054 & & $711(69.4)$ & 712 (69.5) & -0.002 & \\
\hline T3 & $214(18.6)$ & 271 (17.7) & -0.024 & & $193(18.8)$ & $195(19.0)$ & -0.005 & \\
\hline TX & $4(0.3)$ & $1(0.1)$ & -0.062 & & - & - & - & \\
\hline
\end{tabular}

Abbreviations: AJCC, American Joint Committee on Cancer; Sdiff, standardized difference.

*P value for chi-square test or Fisher's exact test.

tP value for McNemar's test or general McNemar's test.

$\ddagger$ The 6th Edition of the AJCC Cancer Staging Manual.

§lsotope, implant or the combination. 


\begin{tabular}{|c|c|c|c|c|c|c|c|c|}
\hline \multirow[t]{2}{*}{ Variable } & \multicolumn{4}{|c|}{ Original Data Set } & \multicolumn{4}{|c|}{ Matched Data Set } \\
\hline & $\begin{array}{l}\text { Right-sided } \\
(n=1150)\end{array}$ & $\begin{array}{l}\text { Left-sided } \\
(n=1533)\end{array}$ & Sdiff & $P^{*}$ & $\begin{array}{l}\text { Right-sided } \\
(n=1025)\end{array}$ & $\begin{array}{l}\text { Left-sided } \\
(n=1025)\end{array}$ & Sdiff & $\mathrm{Pt}$ \\
\hline \multicolumn{9}{|l|}{ Clinical N stage } \\
\hline NO & $466(40.5)$ & $533(34.8)$ & -0.119 & \multirow[t]{3}{*}{0.005} & $400(39.0)$ & $401(39.1)$ & -0.002 & \multirow[t]{3}{*}{0.507} \\
\hline N1 & $412(35.8)$ & $631(41.2)$ & 0.109 & & $380(37.1)$ & $355(34.6)$ & 0.051 & \\
\hline N2 & $272(23.7)$ & $369(24.1)$ & 0.010 & & $245(23.9)$ & $269(26.2)$ & -0.054 & \\
\hline \multicolumn{9}{|l|}{ Original site } \\
\hline Lung lobe & $892(77.6)$ & $1250(81.5)$ & 0.098 & \multirow[t]{4}{*}{0.007} & 817 (79.7) & 792 (77.3) & 0.059 & \multirow[t]{4}{*}{0.544} \\
\hline Main bronchus & $102(8.9)$ & $140(9.1)$ & 0.009 & & $91(8.9)$ & $113(11.0)$ & -0.072 & \\
\hline Overlapping lesion of lung & $121(10.5)$ & $108(7.0)$ & -0.123 & & $93(9.1)$ & $91(8.9)$ & 0.007 & \\
\hline NOS & $35(3.0)$ & $35(2.3)$ & -0.047 & & $24(2.3)$ & $29(2.8)$ & -0.031 & \\
\hline \multicolumn{9}{|l|}{ Histology } \\
\hline Adenocarcinoma & $411(35.7)$ & $417(27.2)$ & -0.183 & \multirow[t]{5}{*}{$<0.001$} & $328(32.0)$ & 317 (30.9) & 0.023 & \multirow[t]{5}{*}{0.774} \\
\hline Squamous cell carcinoma & $563(49.0)$ & $882(57.5)$ & 0.171 & & $537(52.4)$ & $540(52.7)$ & -0.006 & \\
\hline Large cell carcinoma & $38(3.3)$ & $66(4.3)$ & 0.052 & & $38(3.7)$ & $38(3.7)$ & 0.000 & \\
\hline Other & $48(4.2)$ & $60(3.9)$ & -0.013 & & $43(4.2)$ & $46(4.5)$ & -0.014 & \\
\hline NSCLC NOS & $90(7.8)$ & $108(7.0)$ & -0.030 & & $79(7.7)$ & $84(8.2)$ & -0.018 & \\
\hline \multicolumn{9}{|l|}{ Lymph node dissection } \\
\hline 1 to 3 removed & $66(5.7)$ & $65(4.2)$ & -0.069 & \multirow[t]{3}{*}{0.106} & $53(5.2)$ & $49(4.8)$ & 0.018 & \multirow[t]{3}{*}{0.811} \\
\hline 4 or more removed & $994(86.4)$ & $1364(89.0)$ & 0.077 & & $898(87.6)$ & 907 (88.5) & -0.027 & \\
\hline None/Unknown & $90(7.8)$ & $104(6.8)$ & -0.040 & & $74(7.2)$ & $69(6.7)$ & 0.019 & \\
\hline \multicolumn{9}{|l|}{ Radiotherapy } \\
\hline None/Unknown & $918(79.8)$ & $1201(78.3)$ & -0.036 & \multirow[t]{3}{*}{0.335} & $811(79.1)$ & 798 (77.9) & 0.031 & \multirow[t]{3}{*}{0.301} \\
\hline Other§ & $4(0.3)$ & $11(0.7)$ & 0.051 & & $2(0.2)$ & $1(0.1)$ & 0.026 & \\
\hline Beam radiation & $228(19.8)$ & $321(20.9)$ & 0.028 & & $212(20.7)$ & $226(22.0)$ & -0.033 & \\
\hline \multicolumn{9}{|l|}{ Chemotherapy } \\
\hline Yes & $555(48.4)$ & $808(52.7)$ & 0.089 & \multirow[t]{2}{*}{0.025} & $501(48.9)$ & $540(52.7)$ & -0.076 & \multirow[t]{2}{*}{0.061} \\
\hline No/Unknown & $595(51.7)$ & 725 (47.3) & -0.089 & & $524(51.1)$ & $485(47.3)$ & 0.076 & \\
\hline \multicolumn{9}{|c|}{ Abbreviations: AJCC, American Joint Committee on Cancer; Sdiff, standardized difference. } \\
\hline \multicolumn{9}{|c|}{ *P value for chi-square test or Fisher's exact test. } \\
\hline \multicolumn{9}{|c|}{ †P value for McNemar's test or general McNemar's test. } \\
\hline \multicolumn{9}{|c|}{ ¥The 6th Edition of the AJCC Cancer Staging Manual. } \\
\hline §lsotope, implant or the col & ination. & & & & & & & \\
\hline
\end{tabular}

\section{Survival}


The median follow-up was 25 months. Cancer-specific survival $(\mathrm{HR}=0.863,95 \% \mathrm{Cl}: 0.771$ to $0.965, \mathrm{P}=0.010)$ and $\mathrm{OS}(\mathrm{HR}=0.875$, $95 \% \mathrm{Cl}: 0.793$ to $0.967, \mathrm{P}=0.008$ ) were significantly superior with left-sided pneumonectomy compared with right-sided pneumonectomy with the 5 -year OS rate of $36.4 \%(95 \% \mathrm{Cl}$ : $33.4-39.6 \%)$ and $40.2 \%(95 \% \mathrm{Cl}: 37.5-43.1 \%)$ for patients received rightsided and left-sided pneumonectomy respectively (eFigure 2). After multivariable adjustment, differences were also significant between left-sided and right-sided pneumonectomy both in cancer-specific survival $(\mathrm{HR}=0.836,95 \% \mathrm{Cl}: 0.746$ to $0.937, \mathrm{P}=0.002)$ and OS (HR $=0.841,95 \% \mathrm{Cl}: 0.760$ to $0.929, \mathrm{P} \otimes 0.001)$ (Table 2). 
Table 2

Prognostic Factors for Overall and Cancer-specific Survival in All Stage I-IIIA NSCLC Patients with Pneumonectomy in Original Data

Set

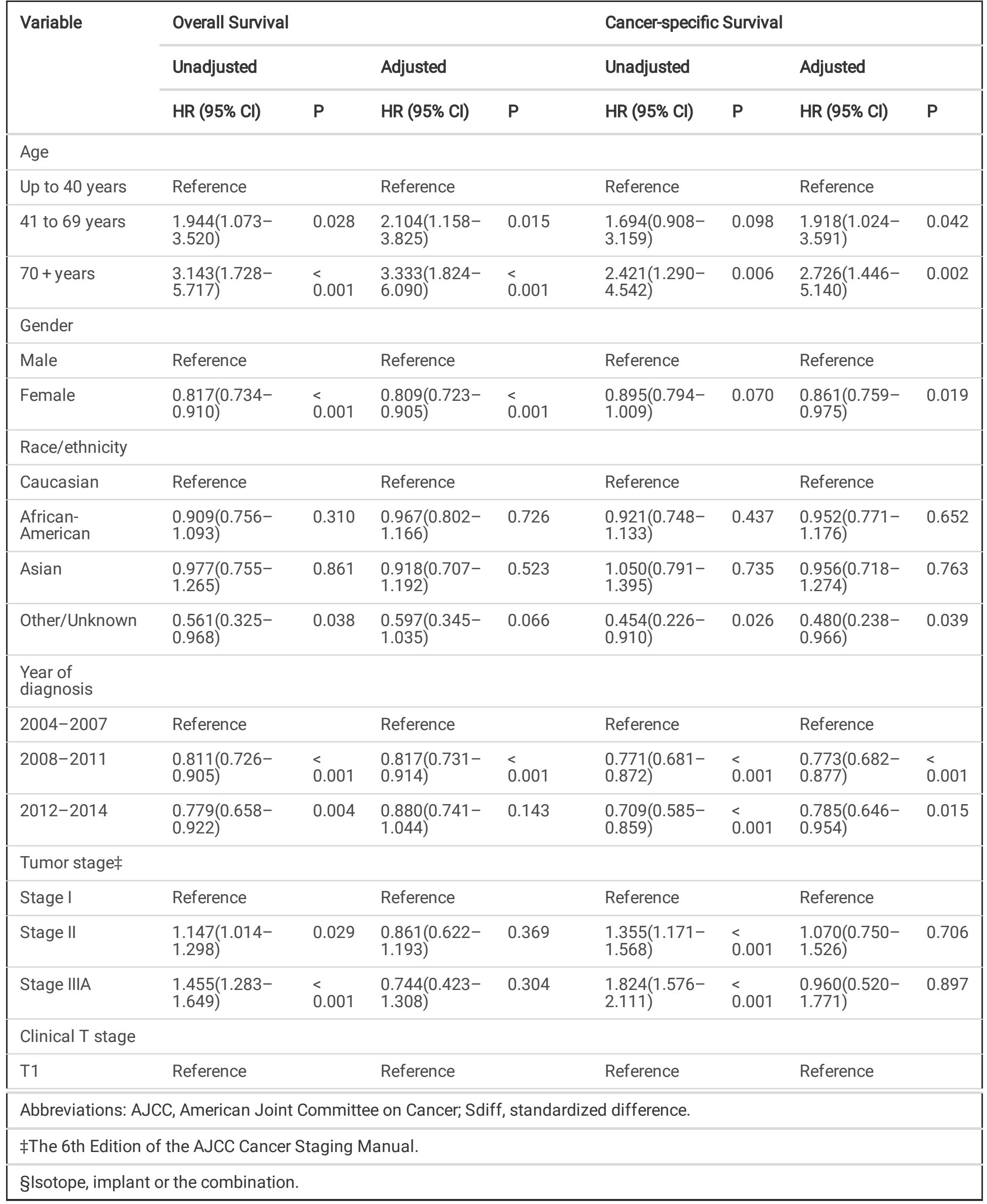




\begin{tabular}{|c|c|c|c|c|c|c|c|c|}
\hline \multirow[t]{3}{*}{ Variable } & \multicolumn{4}{|l|}{ Overall Survival } & \multicolumn{4}{|c|}{ Cancer-specific Survival } \\
\hline & \multicolumn{2}{|l|}{ Unadjusted } & \multicolumn{2}{|l|}{ Adjusted } & \multicolumn{2}{|l|}{ Unadjusted } & \multicolumn{2}{|l|}{ Adjusted } \\
\hline & $\mathrm{HR}(95 \% \mathrm{Cl})$ & $P$ & $\mathrm{HR}(95 \% \mathrm{Cl})$ & $P$ & $\mathrm{HR}(95 \% \mathrm{Cl})$ & $\mathbf{P}$ & $\mathrm{HR}(95 \% \mathrm{Cl})$ & $P$ \\
\hline T2 & $\begin{array}{l}1.210(1.026- \\
1.427)\end{array}$ & 0.023 & $\begin{array}{l}1.267(1.070- \\
1.499)\end{array}$ & 0.006 & $\begin{array}{l}1.443(1.179- \\
1.767)\end{array}$ & $\begin{array}{l}< \\
0.001\end{array}$ & $\begin{array}{l}1.484(1.207- \\
1.824)\end{array}$ & $<.001$ \\
\hline Т3 & $\begin{array}{l}1.347(1.113- \\
1.632)\end{array}$ & 0.002 & $\begin{array}{l}1.723(1.293- \\
2.296)\end{array}$ & $<.001$ & $\begin{array}{l}1.749(1.393- \\
2.196)\end{array}$ & $\stackrel{<}{0.001}$ & $\begin{array}{l}2.002(1.45- \\
2.766)\end{array}$ & $<0.001$ \\
\hline TX & $\begin{array}{l}1.359(0.558- \\
3.309)\end{array}$ & 0.499 & $\begin{array}{l}1.256(0.511- \\
3.090)\end{array}$ & 0.619 & $\begin{array}{l}1.264(0.401- \\
3.984)\end{array}$ & 0.688 & $\begin{array}{l}0.946(0.297- \\
3.008)\end{array}$ & 0.925 \\
\hline \multicolumn{9}{|l|}{ Clinical N stage } \\
\hline NO & Reference & & Reference & & Reference & & Reference & \\
\hline N1 & $\begin{array}{l}1.175(1.046- \\
1.320)\end{array}$ & 0.006 & $\begin{array}{l}1.593(1.152- \\
2.202)\end{array}$ & 0.005 & $\begin{array}{l}1.308(1.143- \\
1.497)\end{array}$ & $\begin{array}{l}< \\
0.001\end{array}$ & $\begin{array}{l}1.454(1.024- \\
2.063)\end{array}$ & 0.036 \\
\hline N2 & $\begin{array}{l}1.484(1.307- \\
1.684)\end{array}$ & $<.001$ & $\begin{array}{l}2.370(1.399- \\
4.016)\end{array}$ & 0.001 & $\begin{array}{l}1.780(1.542- \\
2.054)\end{array}$ & $<.001$ & $\begin{array}{l}2.165(1.225- \\
3.826)\end{array}$ & 0.007 \\
\hline \multicolumn{9}{|l|}{ Original site } \\
\hline Lung lobe & Reference & & Reference & & Reference & & Reference & \\
\hline Main bronchus & $\begin{array}{l}0.889(0.745- \\
1.062)\end{array}$ & 0.195 & $\begin{array}{l}0.894(0.746- \\
1.072)\end{array}$ & 0.227 & $\begin{array}{l}0.915(0.749- \\
1.117)\end{array}$ & 0.381 & $\begin{array}{l}0.931(0.759- \\
1.141)\end{array}$ & 0.492 \\
\hline $\begin{array}{l}\text { Overlapping } \\
\text { lesion of lung }\end{array}$ & $\begin{array}{l}0.888(0.738- \\
1.069)\end{array}$ & 0.209 & $\begin{array}{l}0.852(0.706- \\
1.027)\end{array}$ & 0.094 & $\begin{array}{l}0.958(0.782- \\
1.175)\end{array}$ & 0.684 & $\begin{array}{l}0.909(0.739- \\
1.118)\end{array}$ & 0.367 \\
\hline NOS & $\begin{array}{l}1.507(1.148- \\
1.979)\end{array}$ & 0.003 & $\begin{array}{l}1.508(1.146- \\
1.986)\end{array}$ & 0.003 & $\begin{array}{l}1.467(1.074- \\
2.006)\end{array}$ & 0.016 & $\begin{array}{l}1.440(1.051- \\
1.974)\end{array}$ & 0.023 \\
\hline \multicolumn{9}{|l|}{ Histology } \\
\hline Adenocarcinoma & Reference & & Reference & & Reference & & Reference & \\
\hline $\begin{array}{l}\text { Squamous cell } \\
\text { carcinoma }\end{array}$ & $\begin{array}{l}1.041(0.929- \\
1.165)\end{array}$ & 0.485 & $\begin{array}{l}0.979(0.868- \\
1.104)\end{array}$ & 0.737 & $\begin{array}{l}0.914(0.805- \\
1.037)\end{array}$ & 0.164 & $\begin{array}{l}0.863(0.754- \\
0.988)\end{array}$ & 0.032 \\
\hline $\begin{array}{l}\text { Large cell } \\
\text { carcinoma }\end{array}$ & $\begin{array}{l}1.474(1.155- \\
1.882)\end{array}$ & 0.002 & $\begin{array}{l}1.373(1.072- \\
1.758)\end{array}$ & 0.012 & $\begin{array}{l}1.593(1.231- \\
2.061)\end{array}$ & $\begin{array}{l}< \\
0.001\end{array}$ & $\begin{array}{l}1.469(1.131- \\
1.907)\end{array}$ & 0.004 \\
\hline Other & $\begin{array}{l}1.348(1.045- \\
1.738)\end{array}$ & 0.021 & $\begin{array}{l}1.337(1.034- \\
1.729)\end{array}$ & 0.026 & $\begin{array}{l}1.329(1.004- \\
1.759)\end{array}$ & 0.046 & $\begin{array}{l}1.302(0.981- \\
1.727)\end{array}$ & 0.067 \\
\hline NSCLC NOS & $\begin{array}{l}1.011(0.830- \\
1.232)\end{array}$ & 0.912 & $\begin{array}{l}0.977(0.799- \\
1.195)\end{array}$ & 0.824 & $\begin{array}{l}0.989(0.794- \\
1.233)\end{array}$ & 0.928 & $\begin{array}{l}0.918(0.733- \\
1.149)\end{array}$ & 0.456 \\
\hline \multicolumn{9}{|l|}{$\begin{array}{l}\text { Lymph node } \\
\text { dissection }\end{array}$} \\
\hline 1 to 3 removed & Reference & & Reference & & Reference & & Reference & \\
\hline $\begin{array}{l}4 \text { or more } \\
\text { removed }\end{array}$ & $\begin{array}{l}0.846(0.685- \\
1.045)\end{array}$ & 0.120 & $\begin{array}{l}0.845(0.682- \\
1.047)\end{array}$ & 0.125 & $\begin{array}{l}0.942(0.732- \\
1.212)\end{array}$ & 0.642 & $\begin{array}{l}0.916(0.709- \\
1.184)\end{array}$ & 0.506 \\
\hline None/Unknown & $\begin{array}{l}0.964(0.737- \\
1.260)\end{array}$ & 0.788 & $\begin{array}{l}0.989(0.754- \\
1.297)\end{array}$ & 0.936 & $\begin{array}{l}1.128(0.827- \\
1.539)\end{array}$ & 0.448 & $\begin{array}{l}1.153(0.842- \\
1.578)\end{array}$ & 0.374 \\
\hline \multicolumn{9}{|c|}{ Abbreviations: AJCC, American Joint Committee on Cancer; Sdiff, standardized difference. } \\
\hline \multicolumn{9}{|c|}{ ¥The 6th Edition of the AJCC Cancer Staging Manual. } \\
\hline & le combir & & & & & & & \\
\hline
\end{tabular}




\begin{tabular}{|c|c|c|c|c|c|c|c|c|}
\hline \multirow[t]{3}{*}{ Variable } & \multicolumn{4}{|l|}{ Overall Survival } & \multicolumn{4}{|c|}{ Cancer-specific Survival } \\
\hline & \multicolumn{2}{|l|}{ Unadjusted } & \multicolumn{2}{|l|}{ Adjusted } & \multicolumn{2}{|l|}{ Unadjusted } & \multicolumn{2}{|l|}{ Adjusted } \\
\hline & $\mathrm{HR}(95 \% \mathrm{Cl})$ & $\mathbf{P}$ & $\mathrm{HR}(95 \% \mathrm{Cl})$ & $\mathbf{P}$ & $\mathrm{HR}(95 \% \mathrm{Cl})$ & $\mathbf{P}$ & $\mathrm{HR}(95 \% \mathrm{Cl})$ & $\mathbf{P}$ \\
\hline \multicolumn{9}{|l|}{ Radiotherapy } \\
\hline None/Unknown & Reference & & Reference & & Reference & & Reference & \\
\hline Other§ & $\begin{array}{l}1.714(0.992- \\
2.961)\end{array}$ & 0.053 & $\begin{array}{l}2.278(1.310- \\
3.962)\end{array}$ & 0.003 & $\begin{array}{l}1.884(1.040- \\
3.415)\end{array}$ & 0.037 & $\begin{array}{l}2.256(1.236- \\
4.118)\end{array}$ & 0.008 \\
\hline Beam radiation & $\begin{array}{l}1.162(1.033- \\
1.306)\end{array}$ & 0.012 & $\begin{array}{l}1.238(1.083- \\
1.416)\end{array}$ & 0.002 & $\begin{array}{l}1.280(1.124- \\
1.457)\end{array}$ & $<.001$ & $\begin{array}{l}1.217(1.050- \\
1.411)\end{array}$ & 0.009 \\
\hline \multicolumn{9}{|l|}{ Chemotherapy } \\
\hline Yes & Reference & & Reference & & Reference & & Reference & \\
\hline No/Unknown & $\begin{array}{l}1.479(1.340- \\
1.633)\end{array}$ & $\begin{array}{l}< \\
0.001\end{array}$ & $\begin{array}{l}1.741(1.555- \\
1.950)\end{array}$ & $<001$ & $\begin{array}{l}1.255(1.123- \\
1.404)\end{array}$ & $\begin{array}{l}< \\
0.001\end{array}$ & $\begin{array}{l}1.577(1.388- \\
1.791)\end{array}$ & $\begin{array}{l}<.001 \\
0.001\end{array}$ \\
\hline \multicolumn{9}{|l|}{ Cancer side } \\
\hline Right-sided & Reference & & Reference & & Reference & & Reference & \\
\hline Left-sided & $\begin{array}{l}0.875(0.0 .793- \\
0.967)\end{array}$ & 0.008 & $\begin{array}{l}0.841(0.760- \\
0.929)\end{array}$ & $<.001$ & $\begin{array}{l}0.863(0.771- \\
0.965)\end{array}$ & 0.010 & $\begin{array}{l}0.836(0.746- \\
0.937)\end{array}$ & 0.002 \\
\hline \multicolumn{9}{|c|}{ Abbreviations: AJCC, American Joint Committee on Cancer; Sdiff, standardized difference. } \\
\hline \multicolumn{9}{|c|}{ ¥The 6th Edition of the AJCC Cancer Staging Manual. } \\
\hline §lsotope, implan & the combination & & & & & & & \\
\hline
\end{tabular}

Patients who had T2 or T3 $(P<0.001)$, had N1 $(P=0.036)$ or N2 $(P=0.007)$, were large cell carcinoma $(P=0.004)$, were diagnosed before $2008(P<0.001)$, were diagnosed older than $40(P<0.042)$, were male $(P<0.019)$, did not receive chemotherapy $(P<0.001)$, received beam radiotherapy $(P=0.009)$ or other radiotherapy $(P=0.008)$ were associated with significant poorer cancer-specific survival in multivariate analysis (Table 2).

After propensity score matching, the propensity score for NSCLC patients who received right-sided and left-sided pneumonia were both $0.566 \pm 0.073$ respectively $(P=0.833)$ (eFigure3). After matching procedure, 2,050 patients were included for further analysis with 1,025 patients in both group (Table 1). Cancer-specific survival $(\mathrm{HR}=0.847,95 \% \mathrm{Cl}: 0.745$ to $0.963, \mathrm{P}=0.011)$ and OS (HR = $0.858,95 \% \mathrm{Cl}$ : 0.768 to $0.959, \mathrm{P}=0.007$ ) were significantly longer with left-side compared with opposite side (Fig. 1).

\section{Adjuvant Therapy}

We further analyzed the impact of adjuvant therapy on survival. Among left-sided pneumonectomy patients, patients received adjuvant therapy $(\mathrm{N}=860)$ significantly prolonged cancer-specific survival (65 versus 44 months, $\mathrm{HR}=1.319,95 \% \mathrm{Cl}$ : 1.134 to $1.534, \mathrm{P}<0.001)$ and $\mathrm{OS}(47$ versus 28 months, $\mathrm{HR}=1.489,95 \% \mathrm{Cl}: 1.305$ to $1.701, \mathrm{P}<0.001)$ than patients received pneumonectomy alone $(\mathrm{N}=673)$ (eFigure 4). Among right-sided pneumonectomy patients, patients received adjuvant therapy ( $\mathrm{N}=$ 582) significantly prolonged OS (36 versus 25 months, $\mathrm{HR}=1.335,95 \% \mathrm{Cl}: 1.152$ to $1.548, \mathrm{P}<0.001$ ) but did not improve cancerspecific survival (46 versus 46 months, $\mathrm{HR}=1.056,95 \% \mathrm{Cl}$ : 0.893 to $1.249, \mathrm{P}=0.522$ ) compared with patients received pneumonectomy alone ( $\mathrm{N}=568$ ) (eFigure 5). After matching procedure, cancer-specific survival ( 67 versus 51 months, $\mathrm{HR}=1.314$, $95 \% \mathrm{Cl}: 1.093$ to $1.579, \mathrm{P}=0.004$ ) and OS (46 versus 30 months, $\mathrm{HR}=1.458,95 \% \mathrm{Cl}: 1.239$ to $1.715, \mathrm{P}<0.001$ ) were also significantly longer for patients received adjuvant therapy $(\mathrm{N}=581)$ compared with patients received left-sided pneumonectomy alone ( $\mathrm{N}=444)$ (Fig. 2). Patients received right-sided pneumonectomy and adjuvant therapy $(\mathrm{N}=525)$ were not associated with superior cancer-specific survival (46 versus 42 months, $\mathrm{HR}=1.112,95 \% \mathrm{Cl}$ : 0.933 to $1.325, \mathrm{P}=0.236$ ) compared with patients 
received pneumonectomy alone $(\mathrm{N}=500)$ although adjuvant therapy could improve OS (36 versus 25 months, $\mathrm{HR}=1.362,95 \% \mathrm{Cl}$ : 1.165 to 1.592 , Pø0.001) (Fig. 3).

Subgroup analysis showed that adjuvant therapy could significantly improve cancer-specific survival and OS for stage II and stage IIIA patients with pneumonectomy in both side (Pख0.05). Adjuvant therapy was not associated with prolonged cancer-specific survival $(P=0.687)$ and OS $(P=0.177)$ for stage I patients with right-sided pneumonectomy. Although adjuvant therapy could increase OS for stage I patents with pneumonectomy in left lung $(P=0.014)$ but no differences of cancer-specific survival were observed $(P=0.273)$.

Subgroup analysis showed that adjuvant chemotherapy could significantly improve cancer-specific survival and OS for all pneumonectomy patients. But radiotherapy was associated with worse OS and cancer-specific survival for patients with right-sided pneumonectomy. The median OS for right-sided pneumonectomy patients received chemotherapy alone, radiotherapy alone, chemoradiotheapy and no adjuvant therapy were 54 months, 12 months, 28 months, and 25 months, respectively $(P<0.001)$ with the median cancer-specific survival of 68 months, 13 months, 32 months, and 46 months respectively $(P<0.001)$. Radiotherapy did not significantly improve OS and cancer-specific survival for left-sided pneumonectomy patients either. The median OS for leftsided pneumonectomy patients received chemotherapy alone, radiotherapy alone, chemoradiotheapy and no adjuvant therapy were 59 months, 33 months, 36 months, and 28 months, respectively $(P<0.001)$ with the median cancer-specific survival of 94 months, 44 months, 44 months, and 44 months respectively $(P<0.001)$.

OS was favorable for left-sided pneumonectomy across clinical subgroups including stage II or IIIA, squamous cell carcinoma, received chemoradiotherapy or radiotherapy (Fig. 4).

\section{Discussion}

To our knowledge, this is the largest and the first population based study using a novel propensity score matching analysis to assess the influence of pneumonectomy side on NSCLC patients survival outcome. This study indicated that pneumonectomy side could be deemed as an important factor when physicians choosing the most optimal treatment strategies.

Recent years, several studies showed that the survival outcome between right and left-sided pneumonectomy was different ${ }^{[6,8]}$. One previous study showed that the perioperative death incidence occurred higher for patients with right-sided pneumonectomy[8]. It has also been indicated that right pneumonectomy was associated with poorer survival outcome and more postoperative complications $[6,13]$. The results in our study were coincident with previous studies, indicating that OS and cancer-specific survival were significantly longer with left-sided pneumonectomy versus that in right side. Several reasons might contribute to this phenomenon. First, right lung is thought to be more vital for lung function. So pneumonectomy in right lung might lead to more loss of alveolar volume, pulmonary reserve reduction, and thus decrease respiratory function. Second, pneumonectomy is disposed to increase pulmonary artery pressure and even cause right-heart failure. Third, lung cancers located in diverse side have different skipping metastasis routes to mediastinal lymph nodes. Finally, the anatomic structures differed between right and left lung. Therefore, pneumonectomy side could be a fundamental prognostic factor and patients received right-sided pneumonectomy might need closer follow up for the risk of perioperative complications.

Previous studies showed the occurrence of bronchopleural fistula after surgery, more advanced pathological stage and older age were the poor prognositc factors for patients with pneumonectomy $[14,15]$. The poor prognostic factors found in our study included patients with $\mathrm{T} 2$ or $\mathrm{T} 3, \mathrm{~N} 1$ or N2, large cell carcinoma, diagnosed before 2008 , diagnostic age older than 40, male, did not receive chemotherapy, and received beam radiotherapy or other radiotherapy in multivariate analysis. It is worthwhile to note that patients did not receive chemotherapy, received beam radiotherapy or other radiotherapy had worse cancer-specific survival, which indicated that chemotherapy was essential for patients with pneumonectomy while the role of radiotherapy was controversial.

It's imperative to investigate the impact of adjuvant therapy in patients with pneumonectomy. One previous study showed that radiotherapy dose was significantly related to higher death incidence owing to cardiopulmonary disease[16]. Another study indicated that radiotherapy might cause higher death rates due to respiratory disease[5]. But several other studies showed that the mortality difference was not significant with regards to peri-operative chemotherapy and radiotherapy [17, 18]. Our study demonstrated that adjuvant therapy could significantly prolong survival for patients received left-sided pneumonectomy but did not

Page 10/15 
improve survival for patients with right-sided pneumonectomy. We also found that adjuvant chemotherapy was associated with significantly improved survival both for left and right-sided pneumonectomy patients. But radiotherapy did not bring survival benefit for patients with pneumonectomy, and even worsen the prognosis of right-sided pneumonectomy patients. The complications in patients with pneumonectomy could cause cardiopulmonary insufficiency and adjuvant radiotherapy might cause further respiratory function deterioration. This might be the main cause of death for patients with pneumonectomy. Moreover, the results in our study demonstrated that adjuvant therapy were not essential for stage I patients with pneumonectomy, which was also in accordance with previous studies.

There were some limitations in this study. First, we used the 6th edition of American Joint Committee on Cancer staging rather than the 7th edition. Because we selected patients in the SEER database from 2004 to 2014 and all patients had 6th staging records in the database. If we chose the 7th version, there would be much missing values. Besides, there was no significant difference between the 6th and 7th edition stages, which had no considerable deviation on the study results. Second, some variables such as surgical quality or RO margin, were not actually available in the SEER database and we were unable to analyze these data.

Parts of these results were presented at the 2018 American Society of Clinical Oncology Annual Meeting (Abstract \#8524).

\section{Conclusions}

Right-sided pneumonectomy was associated with worse survival compared with left-side. Adjuvant therapy could significantly prolong survival among left-sided pneumonectomy patients while adjuvant therapy did not increase cancer-specific survival for patients with right-sided pneumonectomy. Adjuvant chemotherapy could bring significant benefit for patients who received both left and right-sided pneumonectomy, but radiotherapy worsened prognosis for right-sided pneumonectomy. This study indicated that pneumonectomy side could be deemed as an important factor when physicians choosing the most optimal treatment strategies.

\section{List Of Abbreviations}

NSCLCØnon-small cell lung cancer; SEER, Surveillance, Epidemiology, and End Results; NAACCR, North American Association of Central Cancer Registries; ICD-O, International Classification of Diseases for Oncology; OS, overall survival; HR, hazard ratio

\section{Declarations}

\section{Ethics approval and consent to participate}

The study was exempted from ethical review by the Beijing Cancer Hospital. We obtained the data agreement and data files were downloaded directly from the SEER website

\section{Consent for publication}

Each author satisfies the criteria for authorship and all authors have read it and agreed to the submission.

\section{Availability of data and materials}

Data files were downloaded directly from the SEER website

\section{Competing interests}

The Authors Declared No Potential Conflicts of Interest.

\section{Funding}

This study was funded by Science Foundation of Peking University Cancer Hospital (18-02).

\section{Authors' contributions}


Conceptualization, B.J. and ZP.W.; formal analysis, QW.Z.; investigation, B.J., JJ.L., J.Z., MN.W., TT.A., YY.W., ML.Z., X.Y., J.Z., HX.C., YJ.C., JJ.W., XY.Z, and ZP.W; writing-original draft preparation, B.J.; writing-review and editing, B.J.; supervision, ZP.W.; funding acquisition, ZP.W.

\section{Acknowledgments}

We acknowledge SEER ${ }^{\star S}$ tat team for providing patients' information.

\section{References}

1. Siegel RL, Miller KD, Jemal A. Cancer statistics, 2016. CA Cancer J Clin 2016; 66: 7-30.

2. Chen W, Zheng R, Baade PD et al. Cancer statistics in China, 2015. CA Cancer J Clin 2016; 66: 115-132.

3. Travis WD. Pathology of lung cancer. Clin Chest Med 2011; 32: 669-692.

4. Ferguson MK, Karrison T. Does pneumonectomy for lung cancer adversely influence long-term survival? J Thorac Cardiovasc Surg 2000; 119: 440-448.

5. Kim DJ, Lee JG, Lee CY et al. Long-term survival following pneumonectomy for non-small cell lung cancer: clinical implications for follow-up care. Chest 2007; 132: 178-184.

6. Simon C, Moreno N, Penalver R et al. The side of pneumonectomy influences long-term survival in stage I and II non-small cell lung cancer. Ann Thorac Surg 2007; 84: 952-958.

7. Kim AW, Faber LP, Warren WH et al. Pneumonectomy after chemoradiation therapy for non-small cell lung cancer: does "side" really matter? Ann Thorac Surg 2009; 88: 937-943; discussion 944.

8. Martin J, Ginsberg RJ, Abolhoda A et al. Morbidity and mortality after neoadjuvant therapy for lung cancer: the risks of right pneumonectomy. Ann Thorac Surg 2001; 72: 1149-1154.

9. Kopec SE, Irwin RS, Umali-Torres CB et al. The postpneumonectomy state. Chest 1998; 114: 1158-1184.

10. Surveillance, Epidemiology, and End Results (SEER) Program (www.seer. cancer.gov) Research Data (1973-2014), National Cancer Institute, DCCPS,Surveillance Research Program, Surveillance Systems Branch, released March 2017, based on the March 2017 submission. www.seer.cancer.gov. Accessed 23 March 2017.

11. Wingo PA, Jamison PM, Hiatt RA et al. Building the infrastructure for nationwide cancer surveillance and control-a comparison between the National Program of Cancer Registries (NPCR) and the Surveillance, Epidemiology, and End Results (SEER) Program (United States). Cancer Causes Control 2003; 14: 175-193.

12. Surveillance, Epidemiology, and End Results Program. Data use agreement for the 1973-2014 SEER Research Data File. https://seer.cancer.gov/data/access.html\#agreement. Accessed Mar 23, 2017.

13. Kalathiya RJ, Davenport D, Saha SP. Long-term survival after pneumonectomy for non-small-cell lung cancer. Asian Cardiovasc Thorac Ann 2013; 21: 574-581.

14. Speicher PJ, Ganapathi AM, Englum BR et al. Survival in the elderly after pneumonectomy for early-stage non-small cell lung cancer: a comparison with nonoperative management. J Am Coll Surg 2014; 218: 439-449.

15. Alexiou C, Beggs D, Rogers ML et al. Pneumonectomy for non-small cell lung cancer: predictors of operative mortality and survival. Eur J Cardiothorac Surg 2001; 20: 476-480.

16. Machtay M, Lee JH, Shrager JB et al. Risk of death from intercurrent disease is not excessively increased by modern postoperative radiotherapy for high-risk resected non-small-cell lung carcinoma. J Clin Oncol 2001; 19: 3912-3917.

17. Margaritora S, Cesario A, Cusumano G et al. Pneumonectomy with and without induction chemo-radiotherapy for non-small cell lung cancer: short and long-term results from a single centre. Eur Rev Med Pharmacol Sci 2013; 17: 29-40.

18. Gudbjartsson T, Gyllstedt E, Pikwer A, Jonsson P. Early surgical results after pneumonectomy for non-small cell lung cancer are not affected by preoperative radiotherapy and chemotherapy. Ann Thorac Surg 2008; 86: 376-382.

\section{Figures}




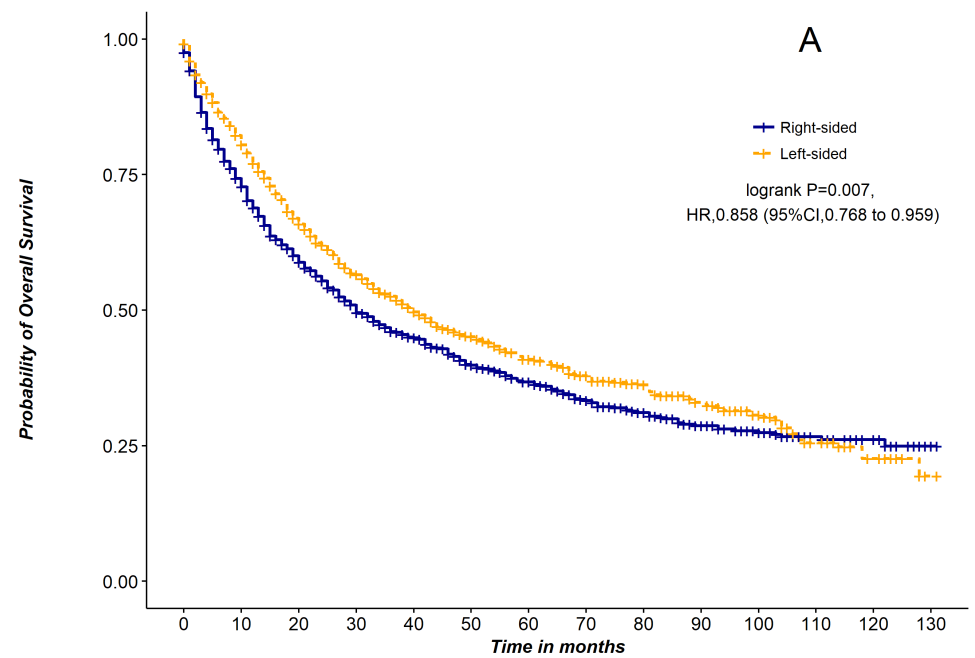

Number at risk

$\begin{array}{lllllllllllllll}\text { Right-sided } & 1025 & 729 & 553 & 420 & 345 & 272 & 230 & 178 & 139 & 104 & 82 & 49 & 26 & 3\end{array}$ $\begin{array}{lllllllllllllll}\text { Left-sided } & 1025 & 797 & 613 & 480 & 373 & 298 & 229 & 188 & 149 & 107 & 78 & 43 & 17 & 2\end{array}$

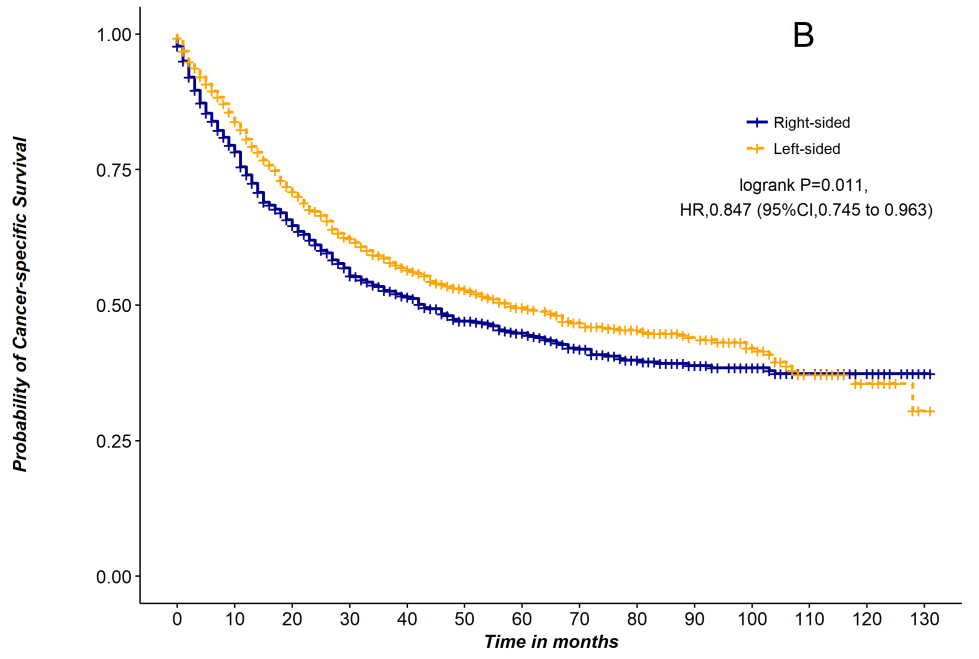

Number at risk

$\begin{array}{lllllllllllllll}\text { Right-sided } & 1025 & 729 & 553 & 420 & 345 & 272 & 230 & 178 & 139 & 104 & 82 & 49 & 26 & 3\end{array}$ $\begin{array}{rllllllllllllll}\text { Left-sided } & 1025 & 797 & 613 & 480 & 373 & 298 & 229 & 188 & 149 & 107 & 78 & 43 & 17 & 2\end{array}$

\section{Figure 1}

Overall Survival and Cancer-specific Survival among Stage I-IIIA NSCLC Patients with Pneumonectomy in the Matched Data Set. (A) Overall Survival. (B) Cancer-specific Survival.
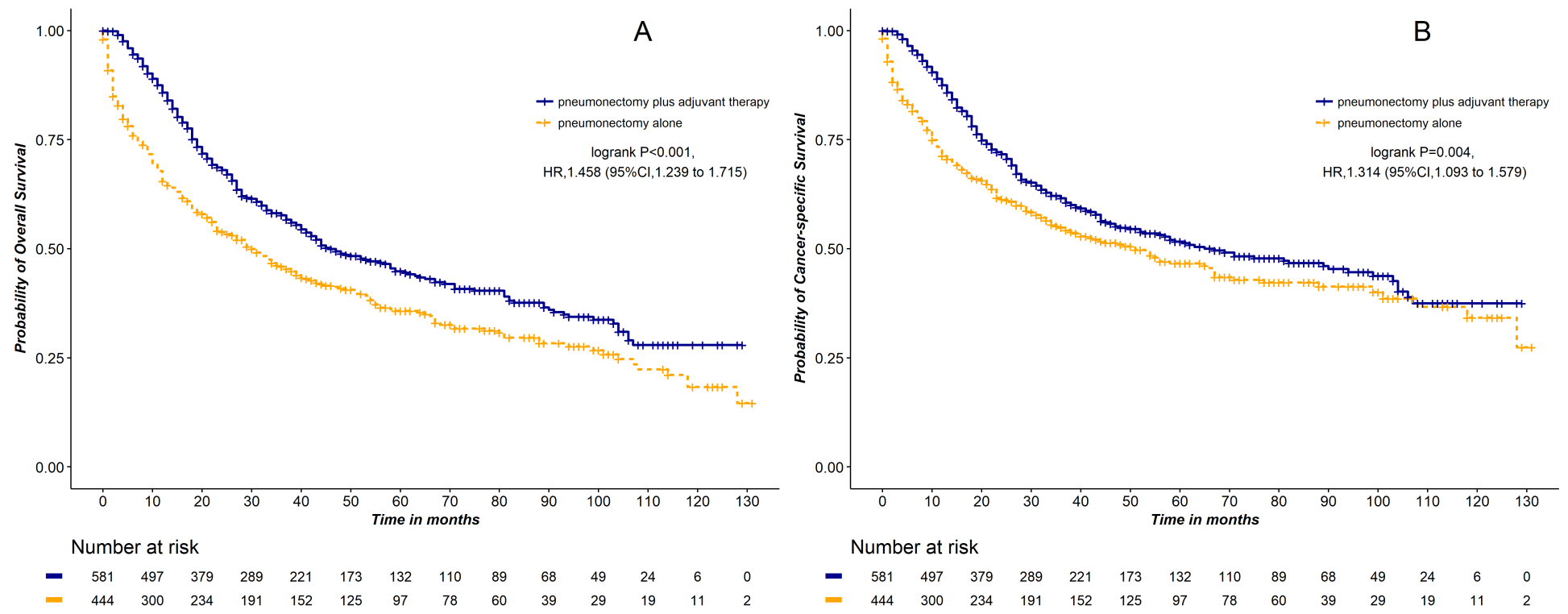

\section{Figure 2}

Overall Survival and Cancer-specific Survival among Stage I-IIIA NSCLC Patients with Pneumonectomy in Left-sided Lung in the Matched Data Set. (A) Overall Survival. (B) Cancer-specific Survival. 

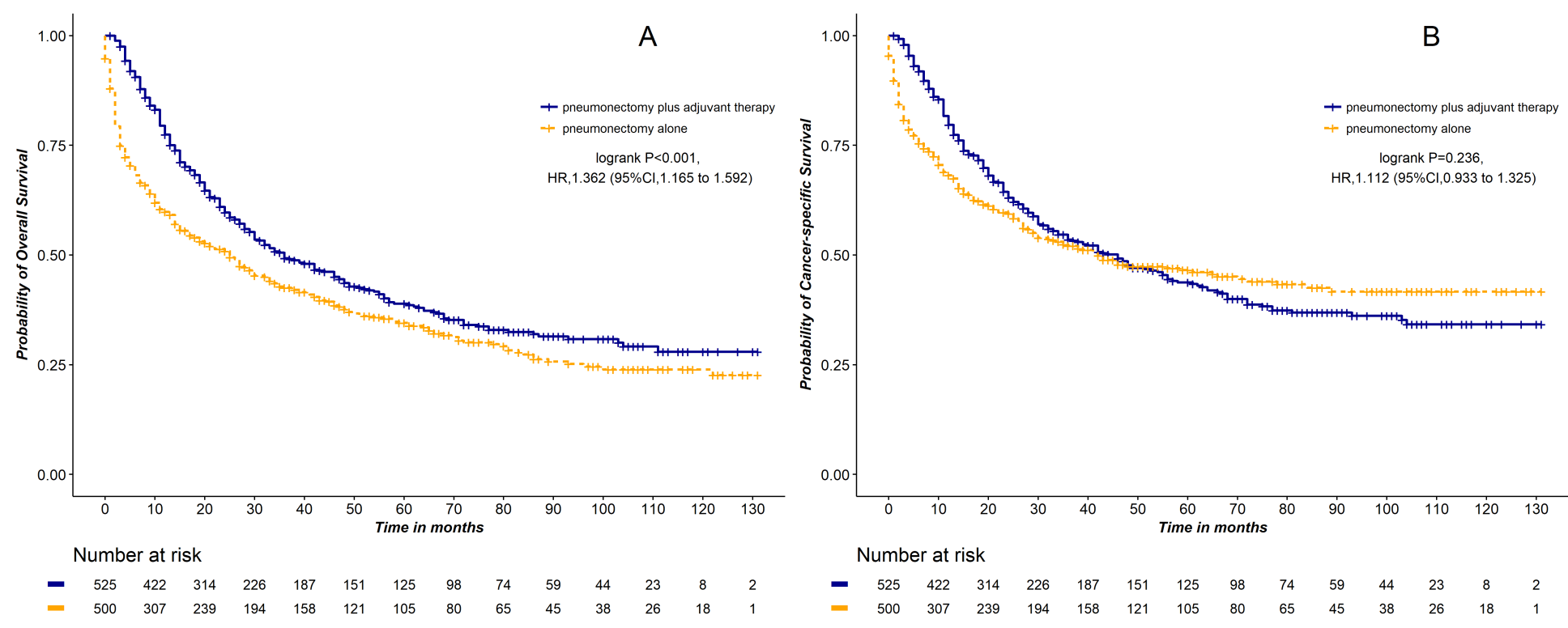

Figure 3

Overall Survival and Cancer-specific Survival among Stage I-IIIA NSCLC Patients with Pneumonectomy in Right-sided Lung in the Matched Data Set. (A) Overall Survival. (B) Cancer-specific Survival.

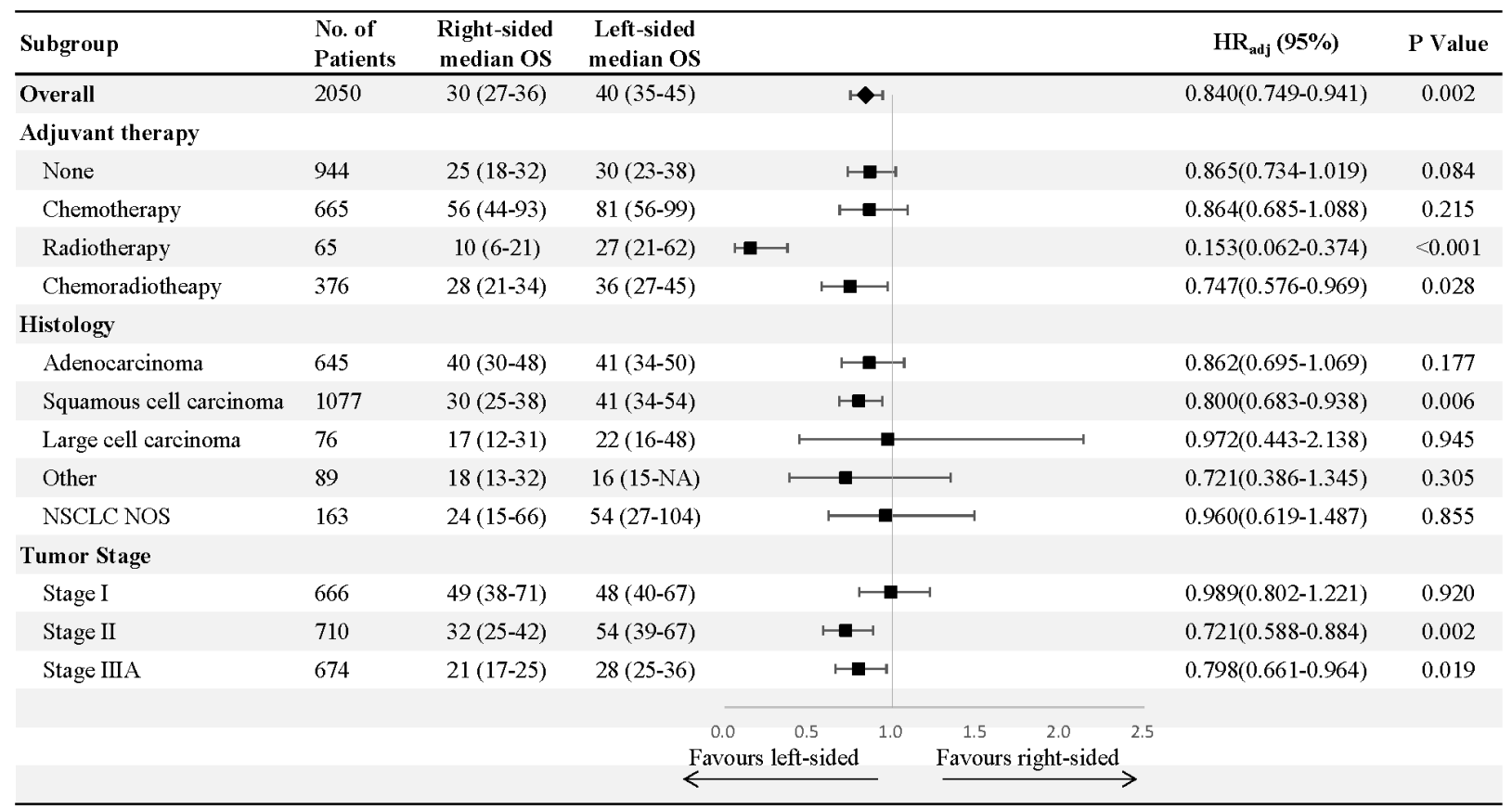

\section{Figure 4}


Overall Survival by subgroup among Stage I-IIIA NSCLC Patients with Pneumonectomy in Right-sided and Left-sided Lung in the Matched Data Set.

\section{Supplementary Files}

This is a list of supplementary files associated with this preprint. Click to download.

- supplementmaterial.docx 